\title{
Editorial: Advances and Obstacles in Contemporary Nonverbal Communication Research
}

\author{
Miles L. Patterson ${ }^{1 *}$, Norah E. Dunbar ${ }^{2}$, Marianne Schmid Mast ${ }^{3}$ and \\ J. M. Fernandez-Dols ${ }^{4}$ \\ ${ }^{1}$ Department of Psychological Sciences, University of Missouri-St. Louis, St. Louis, MO, United States, ${ }^{2}$ Department of \\ Communication, University of California, Santa Barbara, Santa Barbara, CA, United States, ${ }^{3}$ Department of Organizational \\ Behavior, Faculty of Business and Economics, University of Lausanne, Lausanne, Switzerland, ${ }^{4}$ Facultad de Psicología, \\ Universidad Autónoma de Madrid, Madrid, Spain
}

Keywords: nonverbal communication, facial expression, culture, technology, deception

\section{Editorial on the Research Topic}

\section{Advances and Obstacles in Contemporary Nonverbal Communication Research}

For centuries, speculation about the meaning and impact of nonverbal behavior has been common in literature, philosophy, and science (see Knapp, 2006 for a historical review). In the latter half of the nineteenth century, Darwin's 1872 The expression of the emotions in man and animals work was particularly instrumental in focusing attention on expressive behavior. Nevertheless, sustained and systematic empirical research on nonverbal communication was not widespread until the middle of the twentieth century. Examples of its diverse roots can be found in anthropology (Birdwhistell, 1955, 1970; Hall, 1959, 1966), sociology (Goffman, 1959, 1963), and psychology (Sommer, 1959,

OPEN ACCESS

Edited and reviewed by: M. Teresa Anguera, University of Barcelona, Spain

${ }^{*}$ Correspondence:

Miles L. Patterson

miles_patterson@umsl.edu

Specialty section:

This article was submitted to Personality and Social Psychology, a section of the journal

Frontiers in Psychology

Received: 26 June 2021

Accepted: 13 July 2021

Published: 12 August 2021

Citation:

Patterson ML, Dunbar NE, Mast MS and Fernandez-Dols JM (2021)

Editorial: Advances and Obstacles in

Contemporary Nonverbal

Communication Research.

Front. Psychol. 12:731334.

doi: 10.3389/fpsyg.2021.731334
1962; Exline, 1963; Ekman, 1964, 1965). Since that time, literally tens of thousands of articles and hundreds of scholarly books have expanded our knowledge of the nonverbal communication and prompted new and interesting questions about its scope and functions. This acceleration of publications, especially in recent years, provides an appropriate opportunity to examine the current landscape of nonverbal communication research and to provide an outlook into future areas and topics.

In laying the foundation for our "Advances and Obstacles" issue, it is worth noting some of the important topics addressed in current research. For example, we are learning more about the accuracy of pervasive automatic judgments of others' appearance and behavior (Todorov, 2017; Murphy et al., 2019). But automatic judgments can also facilitate prejudice and discrimination, as studies of implicit bias show (Richeson and Shelton, 2005). The long-held view that facial expressions necessarily reflect underlying emotions (Ekman, 1982) is now being challenged. One alternative view proposes that facial behaviors are adaptive and adaptable tools for social influence, rather than universal uniform expressions of basic emotions (Crivelli and Fridlund, 2018). The relative merits of these opposing views also have relevance for understanding nonverbal communication in a variety of settings, including the justice system (e.g., detecting deception), policy decisions, national security, and clinical settings (Denault et al., 2020). Research on cultural differences in nonverbal communication provides insight into cultural dynamics and is relevant for reducing inter-group conflict and facilitating cooperation (Matsumoto and Hwang, 2016). Exciting recent work in behavioral neuroscience examines the neural correlates of nonverbal communication (e.g., Jacob et al., 2014, Lindenberg et al., 2012; Arioli and Canessa, 2019).

In the present digital age, rapidly-evolving communication technologies might seem to displace the more mundane role of face-to-face nonverbal communication in everyday life. The continuing 
expansion of social media, artificial intelligence systems, virtual reality, and social robots, however, is not replacing, but rather extending nonverbal communication to new platforms (see also von der Pütten et al., 2010; Hasler and Friedman, 2012; Küster et al., 2015; Patterson, 2019; Blunden and Brodsky, 2021). As a result, this is a time of expanding research and theory into new domains. Nevertheless, the opportunities provided by the new technologies must be weighed against the ease of spreading misleading and deceptive images that affect our trust in their content (e.g., Tolosana et al., 2020).

Consequently, this is an appropriate time to (1) examine more fully the questions driving current research and theory, (2) weigh the obstacles to a broader understanding of nonverbal communication, and (3) consider the potential opportunities for advancing future research on nonverbal communication. The collection of articles here is testimony to the diversity of nonverbal communication research in addressing these goals.

Many of the 17 articles in this issue focus in some fashion on methodological advances and their potential limitations in new directions for research. Murphy and Hall review the thin-slice method with a particular focus on its reliability and validity in representing sustained behavioral sequences. The article proposes that deciding if and when to employ thin-slice measurement should focus on its broader representativeness for behavior, predictive validity for variables or constructs beyond the sampled behavior, and assessing how the length of the sampled thin-slices affects the accuracy of interpersonal judgments.

Three articles deal with new technologies that include machine learning and the application of algorithms to the scoring and evaluation of nonverbal stimuli. Albohn and Adams applied computer vision algorithms to the structure, color, and texture of faces to predict gender-stereotypic impressions. In addition, the computer impressions were similar to those made by human participants. The broader issue of the opportunities and limitations of machine learning were addressed in two other articles. Burgoon et al. used machine learning and automated analysis to examine the role of dominance-submission, composure-nervousness, and trustmistrust in relational communication. They also discussed the potential benefits of the new techniques in simplifying the study of nonverbal communication. Renier et al. also recognize the utility of applying algorithms in machine learning techniques in analyzing nonverbal behavior. Nevertheless, they caution that automated nonverbal coding can be as biased as human coding and can be limited to the particular context for the behavior.

Several empirical articles focus on a variety of issues related to the encoding and decoding of expressive displays. Bente et al. developed a motion capture and character animation method eliminating cultural and gender appearance cues that can precipitate stereotypic biased judgments. In the absence of visual culture and gender cues, they found that female dyads were rated significantly higher on rapport and that this difference was greater in Arab dyads than in German dyads. Song et al. examined anger and sadness expressions in South Korean and American samples. They found that in both cultures, anger and sadness displays signaled both negative and positive underlying states. Fugate and Franco studied the correspondence between human facial expressions and analogous emoji faces. They found that the majority of emoji faces did not conform to human emotional expressions, even though the anatomical codes for the two types of faces were generally shared. Etcoff et al. investigated the effects of botulinum toxin treatments on the perceptions of pre- and post-treatment smiles. Pre-treatment smiles were rated as more felt, more spontaneous, and happier than posttreatment smiles. Although post-treatment patients were rated as looking younger, they were not judged as more attractive than pre-treatment patients. The effects of tears on visual attention to faces and on subsequent judgments of emotional intensity were the focus of an experimental study by Pico et al. An eye tracking method provided evidence for tears being a magnet for visual attention that, in turn, facilitated perceptions of greater emotional intensity. Ruben et al. addressed the issue of whether technology use enhanced or hindered nonverbal decoding skill. Overall screen time was unrelated to objective measures of decoding skill, but how participants used their screen time was related to decoding skill. Active users (e.g., posting content) performed worse on decoding skill measures, but passive users performed better.

Various issues dealing with authenticity/deception in expressive behavior are the focus of three other articles. Zloteanu and Krumhuber discuss different perspectives on facial displays in the context of increasing evidence contradicting the traditional view that reliable facial muscle movements signal distinct emotional experiences. They discuss spontaneous vs. posed expressions and advocate a functional approach to expressions as neurophysiological states and communicative signals. Vrij and Fisher's article addresses the common assumption that liars display more nervous behaviors than truth tellers. They provide evidence that liars do not show more nervous behaviors. Consequently, observers who focus on such nervous behaviors are likely to do poorly in detecting deception. On a similar theme, Denault discusses the negative consequences of depending on unreliable nonverbal cues for detecting deception. Specifically, in the justice system, judges, and juries are vulnerable to the common, but scientifically discredited, assumption of valid nonverbal indicators of deception. As a result, assessments of witness credibility can be distorted, with detrimental effects on trial outcomes.

The last four articles provide a range of commentaries on approaches to future research. Matsumoto and Hwang advocated for a multimodal approach to research and theory. That is, increased attention to clusters of nonverbal behavior, rather than a single channel at a time, can facilitate our understanding of underlying mental states. Carrard addresses a similar theme of linking interactants' inner preferences and expectations to patterns of nonverbal behavior. That is, nonverbal communication should be viewed as an adaptive process driven by actors' inner characteristics. DeGroot et al. focus on the emerging and important research on the diverse effects of olfaction on a wide variety of interpersonal processes, including identity, emotion, and mate selection. The authors argue that pursuing effectively the wide range of important issues in olfaction requires an integration of the psychology and 
chemistry disciplines into a new field of "sociochemistry." Finally, Kirkwood et al. extend the process of interpersonal synchrony from the nonverbal mimicry between partners to individuals' synchrony with wearable exoskeletons. Recent technological advances in wearable robots are designed to augment a user's strength and mobility. The authors discuss the utility of the Interpersonal Adaptation Theory in facilitating research maximizing human-exoskeleton synchrony.

In conclusion, we hope that this interesting set of articles provides an informative window into some of the diverse issues driving current research on nonverbal communication. The advances in research discussed in many of these articles are often responses to existing obstacles or discrepancies in research. Other articles are focused more on identifying the new obstacles yet to receive attention that, in turn, will stimulate new research.

\section{REFERENCES}

Arioli, M., and Canessa, N. (2019). Neural processing of social interaction: coordinate-based meta-analytic evidence from human neuroimaging studies. Hum. Brain Mapp. 40, 5354-5369. doi: 10.1002/hbm.24627

Birdwhistell, R. L. (1955). Background to kinesics. ETC Rev. General Semant. $13,10-15$.

Birdwhistell, R. L. (1970). Kinesics and Context: Essays on Body Motion Communication. Philadelphia, PA: University of Pennsylvania Press. doi: $10.9783 / 9780812201284$

Blunden, H., and Brodsky, A. (2021). Beyond the emoticon: are there unintentional cues of emotion in email? Pers. Soc. Psychol. Bull. 47, 565-579. doi: $10.1177 / 0146167220936054$

Crivelli, C., and Fridlund, A. J. (2018). Facial displays are tools for social influence. Trends iCognitive Sci. 22, 388-399. doi: 10.1016/j.tics.2018.02.006

Darwin, C. (1872). The Expression of the Emotions in Man and Animals. London: Murray. doi: 10.1037/10001-000

Denault, V., Plusquellec, P., Jupe, L. M., St-Yves, M., Dunbar, N. E., Hartwig, M., et al. (2020). The analysis of nonverbal communication: the dangers of pseudoscience in security and justice contexts. Anuario de Psicol. Jurídica 30, 1-12. doi: 10.5093/apj2019a9

Ekman, P. (1964). Body positions, facial expression and verbal behavior during interviews. J. Abnorm. Soc. Psychol. 68, 295-301. doi: 10.1037/h00 40225

Ekman, P. (1965). Differential communication of affect by head and body cues. J. Pers. Soc. Psychol. 2, 726-735. doi: 10.1037/h0022736

Ekman, P. (1982). Emotion in the Human Face (2nd ed.). New York, NY: Cambridge University Press.

Exline, R. V. (1963). Explorations in the process of person perception: visual interaction in relation to competition, sex, and need for affiliation. J. Pers. 31, 1-20. doi: 10.1111/j.1467-6494.1963.tb01836.x

Goffman, E. (1959). The Presentation of Self in Everyday Life. Garden City, NY: Anchor.

Goffman, E. (1963). Behavior in Public Places. New York, NY: Free Press.

Hall, E. T. (1959). The Silent Language. New York, NY: Doubleday.

Hall, E. T. (1966). The Hidden Dimension. New York, NY: Doubleday.

Hasler, B. S., and Friedman, D. A. (2012). Sociocultural conventions in avatar-mediated nonverbal communication: a cross-cultural analysis of virtual proxemics. J. Intercultural Commun. Res. 41, 238-259. doi: $10.1080 / 17475759.2012 .728764$

Jacob, H., Brück, C., Domin, M., Lotze, M., and Wildgruber, D. (2014). I can't keep your face and voice out of my head: neural correlates of an attentional bias toward nonverbal emotional cues. Cerebral Cortex 24, 1460-1473. doi: 10.1093/cercor/bhs417

Knapp, M. L. (2006). “An historical overview of nonverbal research," in The Sage Handbook of Nonverbal Communication, eds V. Manusov and M. L. Patterson (Thousand Oaks, CA: Sage Publications), 3-19.

Küster, D., Krumhuber, E., and Kappas, A. (2015). "Nonverbal behavior online: a focus on interactions with and via artificial agents and avatars," in The
Thus, the present issue provides a vehicle for facilitating our understanding of nonverbal communication and appreciating where future research may be headed.

\section{AUTHOR CONTRIBUTIONS}

All of the authors have contributed to this work and approved its publication.

\section{ACKNOWLEDGMENTS}

The authors are grateful for the efforts of all of the contributors to this special issue and for the very helpful comments and insights of the manuscript reviewers.

Social Psychology of Nonverbal Communication, eds A. Kostíc and D. Chadee (London: Palgrave Macmillan), 272-302. doi: 10.1057/9781137345868_13

Lindenberg, R., Uhlig, M., Scherfeld, D., Schlaug, G., and Seitz, R. J. (2012). Communication with emblematic gestures: shared and distinct neural correlates of expression and reception. Human Brain Mapp. 33, 812-823. doi: $10.1002 / \mathrm{hbm} .21258$

Matsumoto, D., and Hwang, H. C. (2016). "The cultural bases of nonverbal communication," in APA Handbook of Nonverbal Communication, eds D. Matsumoto, H. C. Hwang, and M. G. Frank (Washington, DC: American Psychological Association), 77-101. doi: 10.1037/14669-004

Murphy, N. A., Hall, J. A., Ruben, M. A., Frauendorfer, D., Schmid Mast, M., Johnson, K. E., et al. (2019). Predictive validity of thin-slice behavior nonverbal behavior from social interactions. Pers. Soc. Psychol. Bull. 45, 983-993. doi: 10.1177/0146167218802834

Patterson, M. L. (2019). A systems model of dyadic nonverbal communication. J. Nonverbal Behav. 43, 111-132. doi: 10.1007/s10919-018-00292-w

Richeson, J. A., and Shelton, J. N. (2005). Brief report: thin slices of racial bias. J. Nonverbal Behav. 29, 75-86. doi: 10.1007/s10919-004-0890-2

Sommer, R. (1959). Studies in personal space. Sociometry 22, 247-260. doi: $10.2307 / 2785668$

Sommer, R. (1962). The distance for comfortable conversation: a further study. Sociometry 25, 111-116. doi: 10.2307/2786041

Todorov, A. (2017). Face Value: The Irresistible Influence of First Impressions. Princeton, NJ: Princeton University Press. doi: 10.1515/9781400885725

Tolosana, R., Vera-Rodriguez, R., Fierrez, J., Morales, A., and Ortega-Garcia, J. (2020). Deepfakes and beyond: a survey of face manipulation and fake detection. Informat. Fusion 64, 131-148. doi: 10.1016/j.inffus.2020.06.014

von der Pütten, A. M., Krämer, N. C., Gratch, J., and Kang, S.-H. (2010). “It doesn't matter what you are!” Explaining social effects of agents and avatars. Comput. Human Behav. 26, 1641-1650. doi: 10.1016/j.chb.2010.06.012

Conflict of Interest: The authors declare that the research was conducted in the absence of any commercial or financial relationships that could be construed as a potential conflict of interest.

Publisher's Note: All claims expressed in this article are solely those of the authors and do not necessarily represent those of their affiliated organizations, or those of the publisher, the editors and the reviewers. Any product that may be evaluated in this article, or claim that may be made by its manufacturer, is not guaranteed or endorsed by the publisher.

Copyright (c) 2021 Patterson, Dunbar, Mast and Fernandez-Dols. This is an openaccess article distributed under the terms of the Creative Commons Attribution License (CC BY). The use, distribution or reproduction in other forums is permitted, provided the original author(s) and the copyright owner(s) are credited and that the original publication in this journal is cited, in accordance with accepted academic practice. No use, distribution or reproduction is permitted which does not comply with these terms. 